\title{
Compenser la destruction de zones humides. Retours d'expérience sur les méthodes et réflexions inspirées par le projet d'aéroport de Notre-Dame-des-Landes (France)
}

Véronique de Billy ${ }^{1}$, Julien Tournebize ${ }^{2}$, Geneviève Barnaud ${ }^{3}$, Marc Benoît ${ }^{4}$, François Birgand $^{5}$, Josette Garnier ${ }^{6}$, Benoît Lesaffre ${ }^{7}$, Christian Lévêque ${ }^{8}$, Ghislain de Marsily ${ }^{9}$, Serge Muller ${ }^{10}$, André Musy ${ }^{11}$, Daniel Zimmer ${ }^{12}$

1 Hydrobiologie, ONEMA, Direction du contrôle des usages et de l'action territoriale, 94300 Vincennes, France

2 Hydrologie, sciences du sol, IRSTEA, Unité de Recherche Hydrosystèmes et bioprocédés, 92761 Antony Cedex, France

3 Écologie et biologie des milieux humides, MNHN, 75005 Paris, France

4 Agronomie des territoires, INRA, Unité ASTER, 88500 Mirecourt, France

5 Hydrologie et biogéochimie des milieux humides, Associate Professor, Biological \& Agricultural Engineering, North Carolina State University, Raleigh, NC 27695-7625, USA

6 Biogéochimie des hydrosystèmes, Sorbonne Universités, Université Pierre-et-Marie-Curie, UMR 7619 METIS, CNRS, EPHE, 75005 Paris, France

7 Hydraulique, vice-président de l'Université Paris-Est, 77455 Marne-la-Vallée, France

8 Écologie des milieux aquatiques, directeur de recherche émérite, IRD, président de l'Académie d'agriculture de France (2013), 75007 Paris, France

${ }^{9}$ Géologie et hydrologie, Sorbonne Universités, Université Pierre-et-Marie-Curie, UMR 7619 METIS, CNRS, EPHE, 75005 Paris, France

${ }^{10}$ Botanique, MNHN, UMR 7205 ISYEB, CNRS, Université Pierre-et-Marie-Curie, EPHE, Sorbonne Universités, 75005 Paris, France

${ }^{11}$ Écohydrologie, École polytechnique fédérale de Lausanne, 1015 Lausanne-Ecublens, Suisse

${ }^{12}$ Hydrologie, KIC Climat, FCS Campus Paris Saclay, 91190 Saint-Aubin, France

Les zones humides constituent des écosystèmes riches et complexes en raison de leur caractère d'interface permanente entre la terre et l'eau. $\mathrm{Si}$, de plus, elles constituent un enjeu d'aménagement majeur avec des conséquences économiques importantes au plan local, elles deviennent une sorte de «barycentre » des attentes et des pressions de tous les acteurs. Dans ce contexte, la question de la compensation de leur altération ou de leur destruction va bien au-delà d'une question de réglementation administrative ou d'analyse coûts-bénéfices. Elle implique les sciences biologiques, les aspects juridiques et enfin les sciences humaines lato sensu. Dans le cas pris comme support de l'étude et présenté dans cet article, le contexte local joue un rôle important sur trois plans : d'abord, au plan de la sociologie des occupants du lieu, et de ses conséquences sur les formes de dialogue ou de non-dialogue ; ensuite au plan du cadre économique local, avec les problèmes chroniques de sous-emploi ou de déclassement de l'agriculture ; enfin, au plan des effets de l'intervention de nombreux acteurs sur les réseaux sociaux. Les auteurs de l'étude explorent comment les procédures de compensation intègrent ou composent avec les multiples composantes du débat.

La Rédaction

Auteur correspondant : V. de Billy, veronique.debilly@onema.fr 
Mots-clés :

compensation ;

zone humide ; retours d'expérience ; fonctions hydrologiques, biogéochimiques et biologiques ; approche systémique ; écologie des paysages

\section{Keywords:} mitigation; wetlands; lessons learned hydrological, biogeochemical, and biological functions; systemic approach; landscape ecology
Résumé - Début 2013, les auteurs de cet article ont été conjointement sollicités par l’État pour donner un avis sur la méthode de compensation des atteintes aux zones humides proposées pour le projet d'aéroport du Grand Ouest à Notre-Dame-des-Landes en Loire-Atlantique. Cet article fait état des réflexions consécutives à ce travail qui, au-delà du cas abordé, portent sur les méthodes à utiliser pour compenser les atteintes aux zones humides altérées, dégradées ou détruites par un aménagement. Il présente tout d'abord le cadre juridique actuel de la compensation; puis l'expérience internationale acquise principalement aux États-Unis, peu de cas similaires existant en France. Enfin, il propose des recommandations sur les modalités d'évaluation du besoin et de la réponse de compensation des atteintes aux zones humides, en insistant notamment sur l'utilité d'y intégrer le facteur temporel, le risque d'échec, l'écologie du paysage et la valeur patrimoniale de ces milieux. En conclusion, l'attention est portée sur les questions que soulèvent la disponibilité et l'usage des terres de compensation, l'incertitude associée aux méthodes de génie écologique et la difficulté de prévoir les trajectoires de ces écosystèmes restaurés/recréés.

\begin{abstract}
Compensation of wetland losses: feedbacks from the airport project at Notre-Dame-desLandes (France). In early 2013, the authors of this article were asked by the French government to jointly assess the adequacy of the method of wetland degradation mitigation proposed for the "Grand Ouest" airport project at Notre-Dame-des-Landes near Nantes (Loire-Atlantique, France). This paper describes the group's reflections following the collective evaluation which, beyond this particular case, concerns the methods to mitigate wetland degradation (destroyed or impacted) by public works. It presents i) the current legal framework of this mitigation; ii) the acquired international experience, mainly in the United States, as few similar cases exist in France; iii) some recommendations on how to evaluate the mitigation needs of wetland degradation, insisting on the need to integrate the time factor, the risk of failure, the landscape ecology and site heritage value. The conclusions highlight the issues of agricultural land availability and suitability for mitigation, the uncertainty associated with methods of ecological engineering and the difficulty of anticipating the trajectories of the restored / recreated ecosystems.
\end{abstract}

\section{Introduction}

À la demande du préfet de la région Pays de la Loire, préfet de la Loire-Atlantique ${ }^{1}$, les auteurs de cet article ont examiné conjointement début 2013 la méthode de compensation proposée par les deux maîtres d'ouvrage pour la construction de l'aéroport du Grand Ouest à Notre-Dame-des-Landes et de sa desserte routière ${ }^{2}$ (Photo 1). L'objet de cet article est de faire état, avec un peu de recul, des réflexions consécutives à ce travail qui, au-delà du cas abordé, portent sur les méthodes et les actions à mener pour compenser l'altération ou la destruction de zones humides par un " aménagement».

Ce terme s'applique ici à toutes installations, ouvrages, travaux ou activités d'origine anthropique. Nous nous sommes néanmoins focalisés sur le cas particulier d'aménagements impactant ${ }^{3}$ de grandes surfaces concentrées sur un seul site. Nos recommandations ne

\footnotetext{
1 Arrêté préfectoral du 21/12/2012 portant création d'un collège d'experts scientifiques dans le cadre de la procédure d'autorisation au titre de la loi sur l'eau concernant le projet d'aéroport du Grand Ouest.

2 Déposé le 9 avril 2013, le rapport est disponible à l'adresse : http://www.developpement-durable.gouv.fr/IMG/ pdf/Rapport_college_experts.pdf.

3 Le terme « impact » fait référence à la définition du Code de l'environnement et englobe les notions d'altération, de dégradation et de destruction des zones humides.
}

sont de ce fait pas forcément transposables à d'autres types de projets tels que les grandes infrastructures linéaires.

Notre réflexion entre dans le cadre des textes réglementaires définissant les modalités de compensation des atteintes aux zones humides, dont ceux figurant dans le "Schéma directeur d'aménagement et de gestion des eaux Loire-Bretagne » (SDAGE, 2010-2015). Si le Code de l'environnement rappelle les grands principes de la compensation écologique (proportionnalité, équivalence, additionnalité, faisabilité, pérennité, etc.), les maîtres d'ouvrage restent libres de proposer leurs propres méthodes d'évaluation des besoins/réponses de compensation, qu'ils doivent ensuite faire valider par les services de l'État. C'est pourquoi ce travail pourrait alimenter la rédaction d'un «Guide des bonnes pratiques » visant deux objectifs : (i) aider les maîtres d'ouvrage à compenser les impacts de leur projet sur l'environnement, sur la base de méthodes pertinentes et efficaces ; (ii) faciliter l'instruction des projets et assurer leur adéquation avec le cadre juridique des actes administratifs qui les ratifient.

Le terme "zone humidé", introduit en France au début des années 1960, est sujet à de multiples interprétations scientifiques et réglementaires en raison de sa polysémie. En 1971, la convention Ramsar ${ }^{4}$ a adopté une

\footnotetext{
4 Traité international signé en 1971 pour la conservation et l'utilisation durable des zones humides visant à enrayer leur
} 


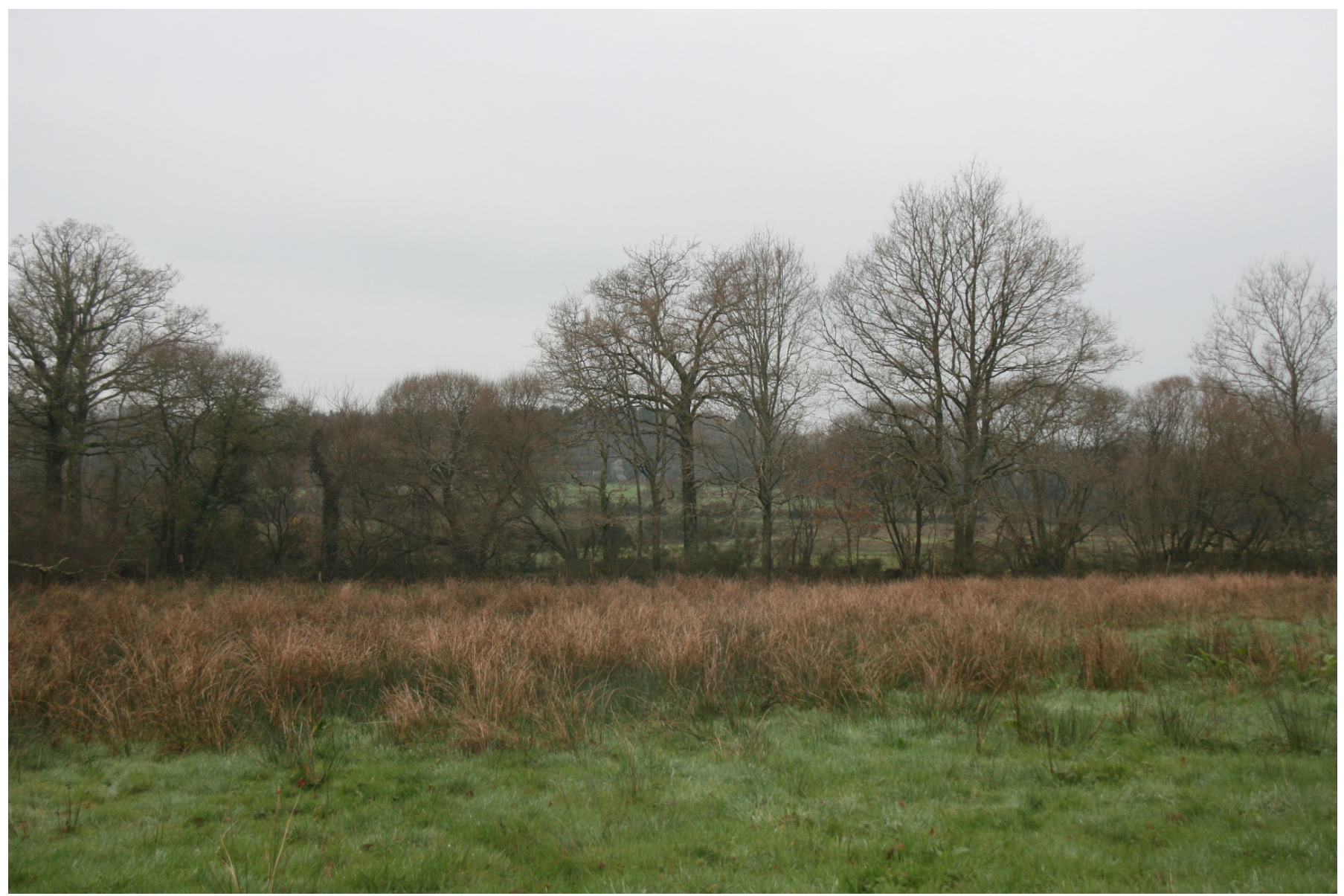

Photo 1. Bocage constitué de prairies humides mésohygrophiles et d'un réseau de haies situé sur le site du futur aéroport de Notre-Dame-des-Landes (source : Serge Muller, MNHN, 14 janvier 2013).

définition assez générale reconnue dans le monde entier, selon laquelle une zone humide est caractérisée par la présence d'eau stagnante ou courante, douce ou salée, que l'écosystème soit d'origine naturelle ou artificielle. Nous avons retenu ici la définition française qui est fondée sur la présence de sols hydromorphes et/ou de plantes hygrophiles et qui exclut les cours d'eau, les plans d'eau et les canaux. Le triptyque "présence d'eau variable dans le temps, de sols caractéristiques et d'espèces adaptées » structure les nombreuses définitions scientifiques existantes, l'accent étant mis sur un ou plusieurs de ces trois critères selon la discipline $\mathrm{d}^{\prime}$ origine de l'auteur (hydrologie, pédologie, biologie). De nouveaux énoncés, issus d'un dialogue entre écologues, biologistes et économistes ont néanmoins émergé, intégrant notamment la problématique des "services écosystémiques » (Costanza et al., 1997).

dégradation ou disparition, en reconnaissant leurs fonctions écologiques ainsi que leur valeur économique, culturelle, scientifique et récréative.

\section{Pourquoi compenser l'impact des aménagements sur les zones humides ?}

En France, depuis des siècles, la nature des zones humides et leur superficie ont été fortement influencées par les usages anthropiques (drainage en vue d'assainir avant mise en culture, imperméabilisation, etc. [Morera, 2011]). Bernard (1994) estime que les deux tiers environ des zones humides métropolitaines françaises ont disparu depuis le début du $X X^{\mathrm{e}}$ siècle dont la moitié en 30 ans (1960-1990). Cette évaluation est proche de celle observée dans d'autres pays européens. Aux États-Unis, la perte de zones humides a été évaluée à $53 \%$ entre 1780 et 1980 (Dahl, 1990).

Terres de transition entre les milieux terrestres et aquatiques, les zones humides comprennent une mosaïque d'habitats adaptés aux gradients d'humidité et parfois de salinité. Elles constituent des foyers de biodiversité, accueillant une flore et une faune souvent rares, protégées et à forte valeur patrimoniale. Elles peuvent être le lieu de pratiques et d'usages agricoles 
extensifs originaux (exemple : élevage de races bovines écossaises ou maraîchines). Leurs fonctions hydrologiques, biogéochimiques et biologiques (régulation des débits, interception des pollutions diffuses, sites privilégiés pour l'alimentation et la reproduction d'espèces animales, etc.) sont à l'origine de "services écosystémiques » que la société juge utile de conserver et de protéger (Amigues et Chevassus-au-Louis, 2011). Ainsi, sur le bassin Loire-Bretagne, le SDAGE précise que «leur préservation, leur restauration et leur recréation, là où elles s'imposent, sont donc des enjeux majeurs. [...]. Les zones humides sont assimilables à des "infrastructures naturelles", y compris celles ayant été créées par l'homme ou dont l'existence en dépend ».

Cependant, si la nécessité de compenser les impacts des aménagements sur ces milieux est désormais admise, les méthodes d'évaluation du besoin et de la réponse de compensation proposées par les maîtres d'ouvrage restent très hétérogènes. Par ailleurs, les retours actuels d'expérience montrent, sur un territoire donné, que les conséquence de la mise en œuvre de mesure de compensation varient selon le type d'aménagement envisagé : pour les grandes infrastructures linéaires (route, ligne ferroviaire, etc.), les besoins de compensation s'étalent sur de longues distances, alors que dans le cas de projets « surfaciques » (aéroport, zone d'activité commerciale, lotissement, zone industrielle, etc.), les besoins de compensation concernent des surfaces géographiquement concentrées à proximité $d u$ projet.

On constate également que toutes les destructions d'écosystème ne sont pas compensables, certains habitats altérés ou détruits ne pouvant être restaurés ou recréés. La connaissance en matière de restauration, de création ou de résilience de certains habitats reste encore lacunaire. Les gains écologiques sont donc très hypothétiques, notamment parce que l'on connaît mal la durée de reconstitution de ces écosystèmes. En outre, la présence et les fonctions de ces habitats dépendent parfois de la connexion, souvent ignorée, avec d'autres habitats, ou d'activités anthropiques révolues, de sorte qu'il faudrait maintenir ou rétablir ces activités pour restaurer ou recréer ces habitats (Lévêque, 2013).

\section{Fondements juridiques et principes méthodologiques de compensation des atteintes aux zones humides}

Dès 1976, le Code de l'environnement introduit en droit français la notion de compensation au sein de la séquence "éviter, réduire, compenser » (ERC), mais cette séquence n'a été clairement définie que dernièrement (MEDDE, 2012a ; CGDD et DEB, 2013). Ainsi, on entend par (i) «éviter », le déplacement géographique ou la modification du projet permettant de supprimer totalement un impact négatif sur l'environnement; (ii) « réduire», la limitation, autant que possible, de la durée, de l'intensité et/ou de l'étendue des impacts du projet sur l'environnement qui ne peuvent pas être complètement évités ; et (iii) «compenser », la réparation en nature des impacts négatifs résiduels significatifs du projet, lorsque les mesures d'évitement et de réduction ne les ont pas supprimés totalement. La compensation intervient donc en dernier lieu, quand les deux autres types de mesures ont atteint leurs limites.

Afin d'évaluer les besoins et les réponses de compensation (Figure 1), le Code de l'environnement a édicté plusieurs principes ${ }^{5}$ fondés notamment sur la proportionnalité (mesures adaptées aux enjeux écologiques associés aux milieux naturels et à l'ampleur des impacts du projet), l'équivalence (nature et fonctions des sites de compensation similaires à celles des sites impactés), l'additionnalité écologique (gain écologique au moins équivalent aux pertes), la faisabilité des actions envisagées (travaux de génie écologique éprouvés et techniquement adaptés aux sites), la proximité géographique et temporelle (pas de dommages irréversibles), l'efficacité (obligations de moyen et de résultat) et la pérennité des mesures mises en œuvre (sécurisation foncière des sites). À ce titre, le respect des objectifs de non-dégradation supplémentaire de l'état des masses d'eau établis par la directive-cadre sur l'eau (2000) sous-entend que les mesures de compensation devraient être recherchées et mises en œuvre en priorité sur les mêmes «masses $\mathrm{d}^{\prime} \mathrm{eau}^{6}{ }^{\star}$ q que celles altérées ou dégradées par le projet. Il est ainsi admis que des écosystèmes (ou leurs fonctions) sont interchangeables entre eux, hypothèse qui fait l'objet de débats. La réglementation évoque néanmoins le fait que tout n'est pas compensable. Si la compensation est complexe, voire impossible dans certains cas, on ne peut en déduire que la réglementation est inapplicable : elle signifie selon nous qu'avant de compenser, il faut éviter ou réduire autant que possible les impacts du projet sur ces milieux.

À l'échelle des grands bassins hydrographiques, les SDAGE reprennent ces principes et proposent différentes méthodes (notamment en termes de ratios de compensation). Le SDAGE Loire-Bretagne prévoit ainsi deux méthodes :

1) La compensation des principales fonctions des zones humides dans le respect des principes d'équivalence et

\footnotetext{
Cf. articles L. 122-3, R. 122-5 § II et R. 122-14 § II du Code de l'environnement.

6 Les masses d'eau de surface correspondent à des tronçons de cours d'eau aux caractéristiques hydromorphologiques homogènes et pour lesquels on peut définir un même objectif d'étatécologique et chimique. Elles servent d'unité d'évaluation de la qualité des eaux au sens de la directive-cadre sur l'eau.
} 


\section{BESOIN DE COMPENSATION}

Évaluation des pertes écologiques (ou« impacts résiduels significatifs»))

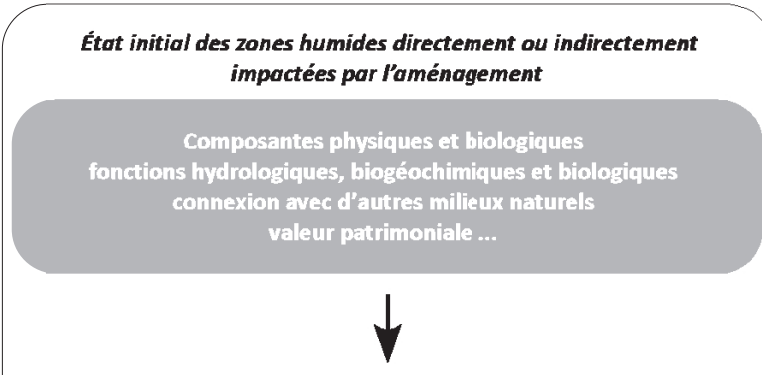

Bilan des mesures d'évitement et de réduction mises en oeuvre en phase chantier et d'exploitotion

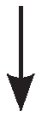

Évaluation des impacts négatifs résiduels significatifs de l'aménagement sur les zones humides, tant sur le plan qualitotif que quantitotif

composantes et fonctions négativement impactées, une fois

les mesures d'évitement et de réduction mises en oeuvre<smiles>CCCCCCCCCCCC</smiles>

Quantification du besoin de compensation

Ajustement des pertes ćcologiques (sur les plans qualitatifs et quantitotifs)

Besoin de compensation réévalué en fonction des enjeux écologiques associés à ces zones humides (rareté, risque de disparition, valeur patrimaniale des habitats, ...)

\section{RÉPONSE DE COMPENSATION}

Évaluation du gain écologique engendrẻ par chaque site de compensation

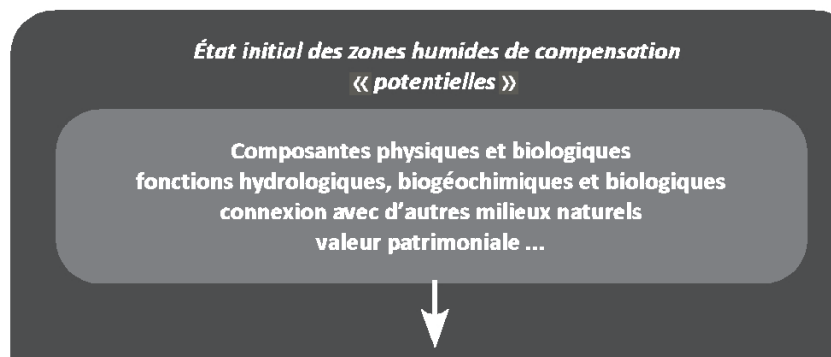

Évaluotion de leur 《trajectoire écologique») (Évolution à moyen/long terme)

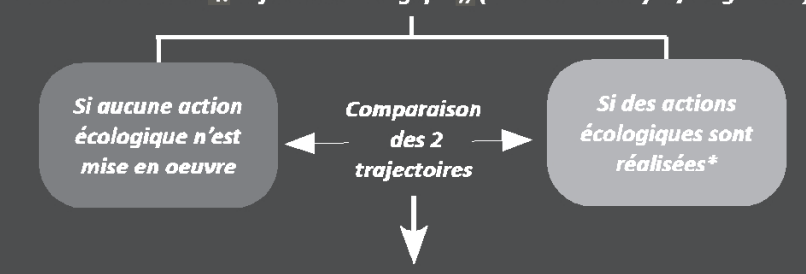

Vérification du respect des principes de compensation : proportionnalité, équivalence, additionnalité écologique et financière, proximité spotiale et temporells, foisabilité, pérennité,

Choix des zones humides de compensation

Quantification de la réponse de compensation

Ajustement des linćaires, surfaces ou volumes à compenser

Réponse de compensotion révaluée en fonction :

du risque d'échec des actions écologiques, du décaloge spotial et

temporel, de (o connexion avec dautres milieux noturels, des modalités de sécurisation foncière, ..
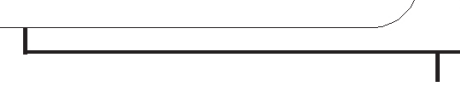

*en complément des actions

3. CONFRONTATION d'ores et déjà menées

BESOIN versUS RÉPONSE DE COMPENSATION

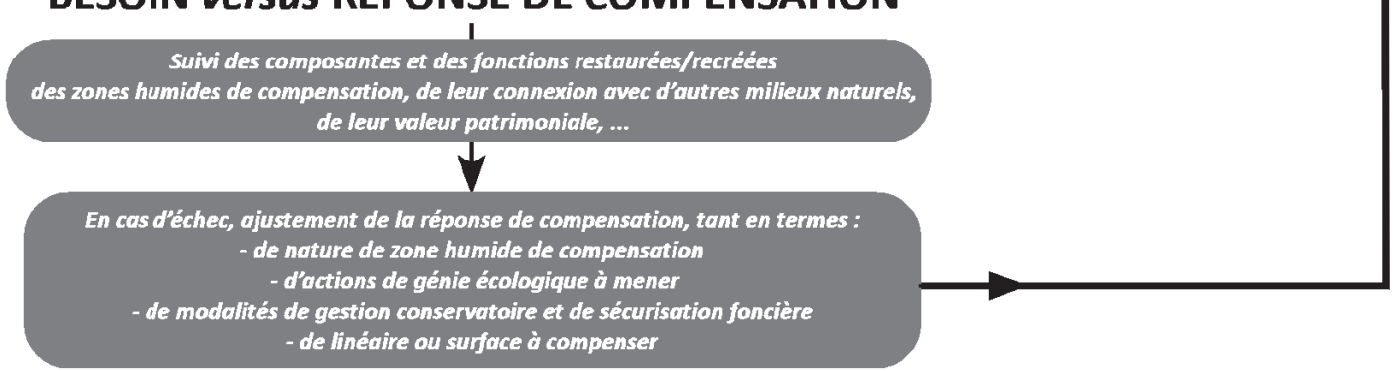

Fig. 1. Schéma de principe illustrant les étapes d'évaluation des besoins et de la réponse de compensation. 
de proximité spatiale (au sein du même bassin versant, de la même "masse d'eau »). Cette approche fonctionnelle nécessite d'identifier les fonctions hydrologiques, biogéochimiques et biologiques des milieux impactés qu'il convient ensuite de restaurer/recréer ailleurs. Elle sert de " garde-fou » afin d'éviter de compenser la destruction de prairies humides par la création de mares, par exemple. Aucun ratio n'étant imposé dans ce cas, il est possible de réduire les surfaces à compenser tout en remédiant, autant que possible, à la perte de certains habitats, fonctions et services rendus par les zones humides sur un territoire donné.

2) À défaut, la compensation calculée sur la base d'un ratio surfacique simple, généralement deux unités compensées pour une détruite (en surface). Dans ce cas, la mise en œuvre des mesures ne s'impose pas nécessairement au même bassin versant, mais au plus proche possible, au sein du même département ou au bassin LoireBretagne tout entier. Cette approche est plus simple, plus pragmatique, plus facile à comprendre et à contrôler, mais consommatrice de territoire.

Il n'est pas précisé si ces deux méthodes peuvent être utilisées conjointement, mais le SDAGE Loire-Bretagne incite davantage les maîtres d'ouvrage à utiliser l'approche fonctionnelle. La combinaison de ces deux méthodes pourrait néanmoins être une option à envisager, la compensation surfacique pouvant alors venir compléter une approche fonctionnelle jugée parfois inapplicable. À noter en outre que dans les schémas d'aménagement et de gestion des eaux (SAGE) du bassin Loire-Bretagne, la notion de "plus-value écologique » est parfois ajoutée, les démarches de compensation devant se traduire par un bilan positif à l'échelle des fonctions majeures de l'écosystème.

\section{Retours d'expérience sur les modalités de mise en œuvre des mesures de compensation}

En ce qui concerne la mise en œuvre opérationnelle des mesures de compensation, différentes approches sont utilisées au niveau international (Morandeau et Vilaysack, 2012). Aux États-Unis, celles-ci découlent de la loi de 1972 (Clean Water Act, article 404, Dredge and Fill Permits) qui admet implicitement la substituabilité des écosystèmes. Son application a évolué au fil du temps et en 2002, un plan d'action national (Wetlands Mitigation) est adopté par l'Administration fédérale pour atteindre l'objectif « pas de perte nette » (no net loss) de zones humides. Ce dernier inclut les trois approches suivantes :

- Le permis « individuel» : la conservation et la restauration de zones humides de compensation sont directement réalisées par le maître d'ouvrage qui est alors titulaire d'un permis d'aménager. Ces mesures sont mises en œuvre sur des sites situés à proximité du site impacté.

- Les banques de compensation : la compensation s'inscrit dans un marché similaire à celui du "carbone » (Geniaux, 2002). Lorsqu'une destruction de zone humide est prévisible sur un territoire donné, la banque achète des terres, y restaure des zones humides dites « fonctionnelles », puis demande une accréditation, qui nécessite une validation technico-administrative du projet et l'atteinte d'objectifs fonctionnels temporels évalués par tranches de 20 à $30 \%$ selon les performances de la restauration écologique (Scemama et Level, 2013). Elle vend ensuite ses crédits aux maîtres d'ouvrage qui en ont besoin pour obtenir leur permis d'aménager. Les transactions sont validées par l'agence environnementale, sur la base de la proximité géographique (même bassin versant) et d'une équivalence fonctionnelle des milieux impactés versus compensés. Le nombre de crédits à acquérir est fondé sur un calcul confrontant les pertes aux gains écologiques obtenus par la banque (Quétier et Lavorel, 2011). Cette approche permet d'anticiper la perte de zones humides, mais la compensation effective des fonctions perdues n'est pas garantie.

- La rémunération de remplacement: le maître d'ouvrage crédite un fond de remplacement qui financera ensuite des projets de création, de restauration ou de préservation de zones humides sur des sites choisis par une agence environnementale. Cette approche relativement risquée est devenue plus sûre en 2008 car réglementairement encadrée. Assez similaire aux banques de compensation, les délais entre impact et compensation restent néanmoins longs (l'action de restauration étant postérieure à la destruction) et la compensation est généralement réalisée sur des terrains publics.

Le succès mitigé de ces trois approches et la volonté de disposer des compensations avant le début des travaux ont conduit l'Administration américaine à favoriser les banques de compensation. Jugées pragmatiques et plus sûres quant aux résultats obtenus (notamment en matière de mise en œuvre, de proximité géographique avec le site impacté et de pérennité), elles sont présentées par leurs défenseurs comme une stratégie "gagnantgagnant » pour l'environnement et les propriétaires fonciers (Ruhl et Salzman, 2006). Elles présentent néanmoins quelques inconvénients, dont des difficultés (i) à respecter le principe d'équivalence entre les pertes et les gains écologiques ; et (ii) à maîtriser la trajectoire de ces écosystèmes en raison d'un défaut de conception de la mesure compensatoire ou de l'échec du génie écologique mis en œuvre ou de la réalisation effective des suivis (Salzman et Ruhl, 2005 ; Quétier et al., 2012).

Concernant l'efficacité des actions écologiques menées pour restaurer les zones humides, des retours d'expérience sont désormais disponibles sur plusieurs continents (Madsen et al., 2010, 2011 ; Barnaud et Coïc, 
2011 ; Morandeau et Vilaysack, 2012) et les États-Unis affichent près de 40 ans d'expérience sur le sujet. Il apparaît que les objectifs sont rarement atteints sur le plan fonctionnel (Moreno-Mateos et al., 2012) et que l'objectif «pas de pertes nettes de zones humides » reste difficile à respecter (Maron et al., 2012). Le Committee on Mitigating Wetland Losses (2001) et Turner et al. (2001) estiment que la législation appliquée jusqu'en 2001 a permis de compenser environ $20 \%$ des zones humides détruites, d'où une perte nette de $80 \%$. Les raisons évoquées sont :

- l'inconsistance ou l'absence de mise en œuvre de ces mesures du fait d'un manque de contrôle. Sur 152 permis accordés, seulement $15 \%$ étaient conformes (GAO, 2001, 2005) ;

- les difficultés techniques rencontrées en matière de création/restauration de zones humides. La métaanalyse réalisée par Moreno-Mateos et al. (2012) à partir de 621 projets recensés dans le monde confirme que la restauration effective de ces milieux est souvent lente, incomplète en raison de blocages successifs (techniques et organisationnels), voire impossible pour certains habitats humides (tourbières, prairies oligotrophes ${ }^{7}$, etc.).

En France et à l'étranger, de nombreux suivis d'opérations de restauration de prairies à biodiversité élevée attestent également du caractère très lent et souvent partiel de telles restaurations (Muller et al., 1998 ; Bakker et Berendse, 1999 ; Pywell et al., 2002 ; Vécrin et Muller, 2003 ; Vécrin et al., 2007 ; Joyce, 2014).

En outre, la trajectoire future des sites de compensation reste incertaine, celle-ci résultant $\mathrm{d}$ 'interactions complexes et de facteurs indépendants des travaux de génie écologique mis en œuvre (Zedler et Callaway, 1999). Cette trajectoire pourra ainsi dépendre de la présence à proximité d'habitats "sources", de l'importance de la «banque de graines » dans le sol, de l'étendue de la zone à restaurer, de la nature des échanges avec les eaux de surface ou souterraines et de l'hydropériode (fréquence, durée, intensité et saisonnalité des variations du niveau d'eau [Matthews et Endress, 2008]). De ce fait, la recréation/restauration de fonctions équivalentes à celles des écosystèmes détruits ou impactés est très hypothétique, ce qui donne tout son sens au terme « éviter » dans la séquence ERC (Photo 2).

\section{Règles à respecter relatives aux méthodes de compensation}

Outre le respect des principes évoqués ci-dessus, l'expérience acquise à Notre-Dame-des-Landes conduit à recommander de respecter les règles suivantes :

- Réaliser un état initial des composantes et des fonctions physiques et biologiques des zones humides

\footnotetext{
7 Associations végétales caractéristiques de sols pauvres en éléments nutritifs et humides.
}

impactées par l'aménagement et des zones humides de compensation, fondé sur un protocole homogène et territorialement représentatif du fonctionnement de ces écosystèmes (Fig. 1). L'objectif est d'estimer l'ensemble des impacts de l'aménagement sur ces milieux, d'en déduire les besoins de compensation et les territoires mobilisables à ce titre, et $d^{\prime}$ anticiper les suivis à mettre en œuvre.

- Justifier le choix de la méthode d'évaluation du besoin et de la réponse de compensation sur la base d'un argumentaire scientifiquement fondé et objectif, élaboré à partir de retours d'expérience comparables à la situation rencontrée. Cette méthode doit rester simple et intelligible pour le public et les parties prenantes, afin notamment qu'ils puissent: (i) prévoir à l'avance les conséquences de ces mesures sur le territoire; (ii) suivre et contrôler les mesures proposées.

- Si une méthode fonctionnelle est retenue, prendre les zones humides impactées et les zones de compensation puis évaluer et comparer : (i) leurs fonctions biologiques intrinsèques (richesse et diversité en habitats et en espèces, rôle en termes de site privilégié pour le repos, l'abri, l'alimentation ou la reproduction de la faune) et extrinsèques (connectivité écologique et continuité paysagère de ces zones avec les autres milieux naturels situés à proximité) ; (ii) leurs fonctions biogéochimiques et hydrologiques (associées à l'hydropériode et à la composition des sols) ; (iii) l'évolution (ou trajectoire) à long terme de ces milieux, avec ou sans action écologique ; et (iv) la valeur patrimoniale de ces paysages, même si ce dernier point n'est pas formellement explicité dans la réglementation.

- Faire preuve d'un certain pragmatisme et de réalisme. Ainsi, la copie conforme de ce qui a été détruit ne saurait être un objectif affiché raisonnable, même en déployant les meilleures techniques disponibles de génie écologique. Il est plus réaliste de se fixer pour objectif " pas de perte nette de fonctions écologiques ", en intégrant des coefficients d'ajustement du besoin et de la réponse de compensation (Fig. 1), dont notamment :

(i) le risque d'échec des actions écologiques à mener, qui est d'autant plus élevé que l'objectif de restauration est ambitieux (Mitsch et Jørgensen, 2004). Ce risque peut être quantifié (Robb, 2002; Moilanen et al, 2009) au regard de la nature et de l'état des sites de compensation avant travaux, des travaux de génie écologique mis en œuvre et des objectifs à atteindre ;

(ii) le temps nécessaire à la restauration effective des milieux dégradés (Bendor et al., 2009), qui varie selon le type d'habitats concerné : de l'ordre de trois ans pour une mare à quelques dizaines d'années pour une haie ou des zones humides d'eau douce herbacées, boisées ou tourbeuses, voire à jamais dans certains cas particuliers (prairie humide oligotrophe [cf. Mitsch et Wilson, 1996]). C'est un exercice difficile d'autant qu'une partie des 


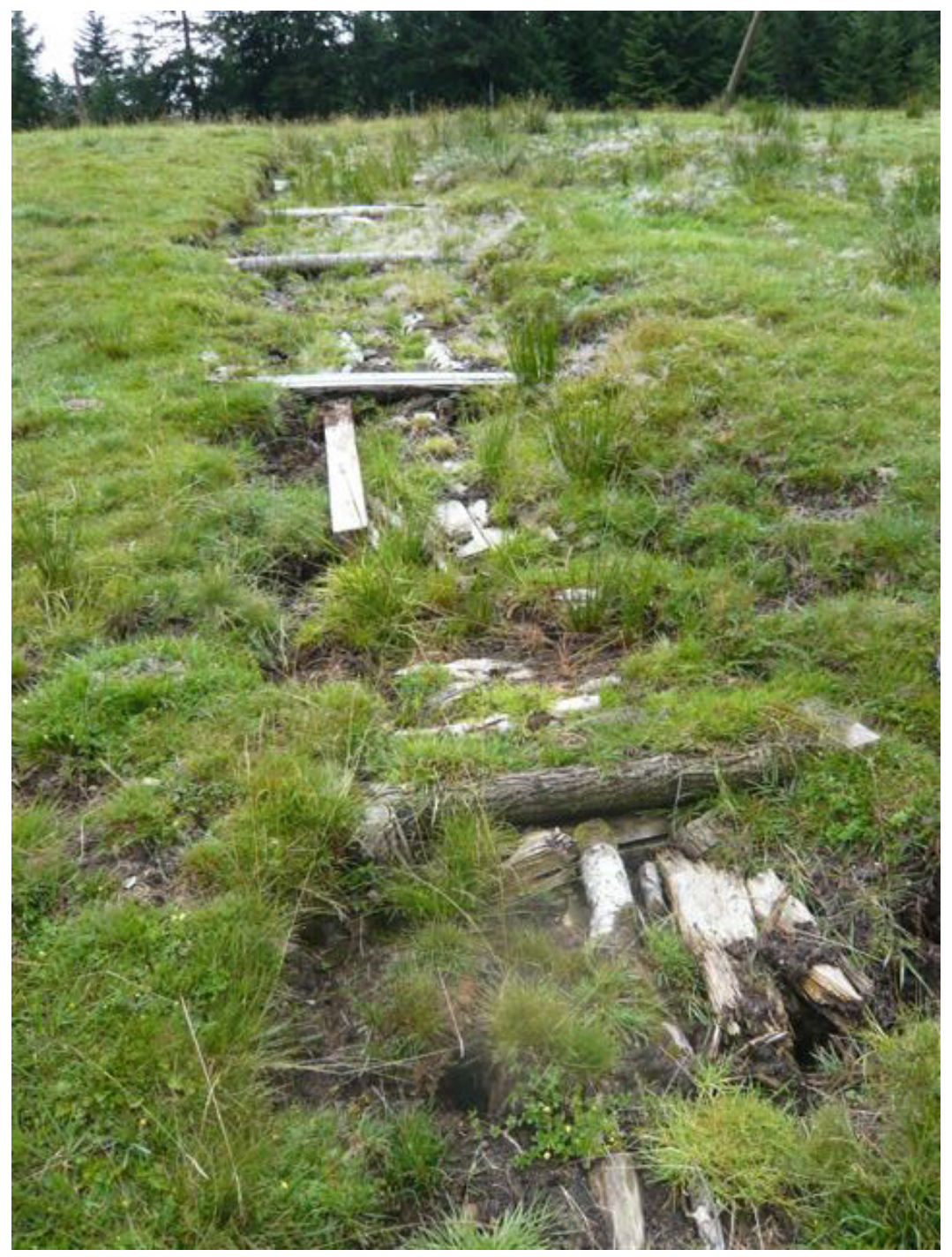

Photo 2. Échec partiel de la restauration d'une tourbière du fait de travaux de génie écologique inappropriés au regard de la pente, des ruissellements superficiels et de l'érosion des sols qui en résulte (source : Thierry Tico, Onema, 9 août 2007).

incertitudes réside dans la méconnaissance de la dynamique temporelle naturelle de ces milieux. Il est toutefois possible d'utiliser les travaux scientifiques existants, les retours d'expérience disponibles et les cycles biologiques des espèces présentes ou susceptibles de recoloniser les zones humides restaurées ;

(iii) la complexité du paysage (ou approche systémique) et la valeur patrimoniale des zones humides.

Ces considérations doivent permettre de pondérer les surfaces à compenser sur la base de ratios surfaciques supérieurs à 1 .

- Adapter les modalités de sécurisation foncière des sites de compensation au temps nécessaire à la restauration/recréation de ces milieux et mettre en place les mesures le plus tôt possible, de préférence avant le démarrage des travaux.
- Garder en mémoire que les zones humides peuvent, dans le cas général, être des systèmes modifiés, évoluant sous l'influence de contraintes naturelles ou anthropiques (Lévêque et Van der Leeuw, 2003). Il faut alors anticiper l'évolution possible de ces milieux, même si la démarche est délicate dès lors qu'elle résulte de changements climatiques ou sociétaux (modification du régime hydrologique, de la politique agricole, etc.).

\section{Mise en œuvre d'une approche systémique}

La présence des zones humides et leurs fonctions $s$ 'inscrivent dans un contexte spatiotemporel plus large d'écologie des paysages, constitués par une mosaïque d'habitats en interaction (Forman et Godron, 1986). En effet, la structure et l'organisation du paysage jouent un 
rôle crucial dans le fonctionnement des écosystèmes, parfois appelés écocomplexes (Blandin et Lamotte, 1984). Selon l'échelle spatiale considérée, des synergies à l'origine de nouvelles fonctions ou d'un renforcement des fonctions liées à l'un des milieux peuvent se mettre en place (Whigham, 1999). Ainsi, des relations existent entre diminution de l'hétérogénéité des habitats, perte de richesse spécifique et capacité d'expression de certaines fonctions écologiques, en particulier la résilience et la productivité (Loreau et al., 2003).

Cette notion de connectivité écologique, très liée à celle de paysage, suggère que la disposition spatiale des éléments du milieu et leur contiguïté doivent être prises en compte lors de l'évaluation du besoin et de la réponse de compensation des fonctions impactées. Il convient en effet de développer cette approche systémique qui considère les fonctions des zones humides dans leur ensemble et en interaction, plutôt que de chercher à les compenser séparément. Cela nécessite de raisonner à l'échelle du bassin versant en tant qu'hydroécosystème avant de passer à celle de la zone humide et de ses fonctions intrinsèques.

La prise en compte, dans les opérations de restauration des zones humides, des effets des changements du paysage sur le fonctionnement des écosystèmes et sur la biodiversité, permet de mieux définir le génie écologique à mettre en œuvre (Tiner, 2002). À titre d'exemple, les travaux récents sur la restauration " passive » de têtes de bassin altérées par le piétinement des troupeaux ont montré l'importance de la disposition du réseau de haies (CORPEN, 2007). La capacité de recolonisation des berges par les arbres dépend de la structure du bocage proche ainsi que des pratiques agricoles appliquées dans les parcelles en bordure des ruisseaux (Forget et al., 2013). Envisager ces paysages comme des produits des activités agricoles et forestières à maîtriser sur le long terme est une condition de leur maintien et de la réussite des travaux de génie écologique mis en œuvre au titre de la compensation (Benoît et al., 2012).

\section{Prise en compte de la non-stationnarité des écosystèmes}

Une question fondamentale se pose concernant l'hypothèse de «stationnarité » du milieu, hypothèse généralement implicite. L'état initial et l'évaluation du besoin et de la réponse de compensation ne prennent généralement pas en compte la dynamique temporelle des écosystèmes, et notamment leur variabilité interannuelle. La réglementation en vigueur n'impose pas de considérer ces variations potentielles du milieu (climat, changement des pratiques agricoles, de l'usage du sol, etc.), mais elle demande d'évaluer les effets directs, indirects et induits du projet, à court et à long terme.

Or, les écosystèmes s'inscrivent toujours dans des trajectoires dynamiques. Ils évoluent dans le temps et dans l'espace sous la contrainte des facteurs climatiques, économiques et sociétaux (Barles, 2013). La notion de trajectoire non rectiligne sert, en écologie de la restauration, à bâtir des scénarios intégrant les facteurs majeurs de changement. Elle permet d'intégrer l'existence de séquences, de boucles de rétroaction, de connexions étroites entre processus physiques et écosystémiques, ainsi que d'obstacles éventuels à l'origine de blocages à une étape donnée (Zedler et Callaway, 1999). Les conséquences des opérations de restauration dépendent de l'ampleur des modifications physiques et du type de génie écologique mis en œuvre, des possibilités de reconnexion entre la zone restaurée et les écosystèmes adjacents (recolonisation, reproduction des espèces), du rétablissement effectif de ces interactions biotiques et de la reprise des processus écologiques (Robson et al., 2011). Ainsi, au cours du suivi postcréation ou restauration, les systèmes subiront à la fois les effets des mesures mises en œuvre et ceux des changements globaux en cours. En l'absence de réflexion sur ce sujet, le risque de se trouver dans quelques décennies face à une situation dite «imprévue » n'est pas négligeable, justifiant que les objectifs n'ont pu être atteints du fait de ces changements pourtant attendus ( $c f$. Erwin, 2009 ; pour le programme Explore 2070, voir MEDDE, 2012b).

Même si les textes réglementaires ne le mentionnent pas explicitement, quelle signification peut-on alors donner aux objectifs de restauration des fonctions majeures des zones humides ? Outre les aléas liés au climat, comment estimer les incertitudes concernant la politique agricole et le comportement d'autres acteurs face aux mesures de compensation? Dans l'idéal, les maîtres d'ouvrage devraient, sur la base des connaissances disponibles, proposer quelques scénarios concernant les trajectoires potentielles des zones de compensation, et ce sur la base d'hypothèses climatiques ou sociétales (tendances d'évolutions de l'activité économique, de l'agriculture, de l'urbanisme, des attentes de la société civile en matière d'environnement, de loisirs, etc.).

À cet égard, la mise en place de «sites témoins 》 - à l'extérieur de l'emprise du projet où les effets de ces changements globaux seront observés indépendamment des mesures de compensation - permettant d'identifier et de comprendre ce qui relève du génie écologique ou des autres perturbations est nécessaire. De tels sites pourront servir à réorienter la réponse de compensation. L'objectif est de garantir que celle-ci soit gérée avec une vision à long terme et d'éviter l'attribution des éventuels échecs du génie écologique à un changement dit « imprévu » de l'environnement. 


\section{Prise en compte de la valeur patrimoniale et esthétique des sites impactés}

La notion de valeur patrimoniale, distincte de celle de «patrimoine naturel » au sens de statuts attribués à des espèces, des habitats et des milieux inventoriés et protégés (Lefeuvre, 1990), n'est pas directement intégrée dans la loi sur l'eau de 2006. Inclure le critère de patrimonialité paraît cependant nécessaire car on parle des fonctions et des services des zones humides, tant d'un point de vue écosystémique que sociétal (Millennium Ecosystem Assessment, 2005).

Le citoyen, qui est en dernier ressort le bénéficiaire des efforts que consent la société pour la protection de la nature, attache sans doute autant d'importance à la qualité et à l'authenticité paysagère des territoires compensés qu'à leurs fonctions physiques et biologiques. Les aspects patrimoniaux et esthétiques jouent donc un rôle important dans la motivation des citoyens à protéger ou non ces milieux (Zedler et al., 2012). Rappelons enfin que les autorisations données par les services de l'État aux maîtres d'ouvrage au titre de la « loi sur l'eau » sont soumises à enquête publique. Cette participation aux choix environnementaux est désormais un droit reconnu, depuis son inscription dans la convention $\mathrm{d}^{\prime} \mathrm{Aarhus}^{8}$ et dans la Charte de l'environnement adossée à la Constitution française. Ces orientations militent pour une prise en compte des aspects patrimoniaux dans la compensation. Afin que cette disposition soit effective, la méthode de compensation à retenir se doit d'être aussi simple et transparente que possible, bien que la matière soit intrinsèquement complexe. De plus, toute absence de clarté rend très difficiles le suivi opérationnel ultérieur et le contrôle des mesures de compensation.

\section{Recommandations relatives à la mise en œuvre des mesures de compensation}

L'identification du besoin et de la réponse de compensation, sur la base de l'étude des fonctions hydrologiques, biogéochimiques et biologiques des zones humides altérées, dégradées ou détruites, d'un côté, et des sites à restaurer ou à recréer au titre de la compensation écologique, de l'autre, doit s'appuyer sur un état initial fiable et complet réalisé à l'échelle du bassin versant puis à celle de chaque site.

Ces fonctions sont généralement interdépendantes. Elles conditionnent le type d'écosystème qui se développe mais n'agissent pas nécessairement aux mêmes

8 Accord international du 25/06/1998 fixant les modalités d'accès à l'information, à la participation du public au processus décisionnel et à l'accès à la justice en matière d'environnement. échelles. Ainsi, il importe de les prendre en compte dans l'ordre suivant :

- Les fonctions hydrologiques (régulation hydraulique par soutien d'étiage, écrêtement des crues, etc.), qui résultent $\mathrm{du}$ fonctionnement hydrogéomorphologique $\mathrm{du}$ bassin versant. Ces dernières conditionnent en effet les fonctions biologiques et biogéochimiques locales. À noter que les travaux de génie écologique nécessaires à la restauration du soutien d'étiage ou à la régulation des crues pourront être différents et ne concerneront pas forcément les mêmes milieux. Dans le cas particulier de Notre-Dame-des-Landes, la compensation de ces fonctions pour les zones humides « de plateau » paraît difficile à proximité des sites dégradés ou détruits, en raison de leur spécificité géographique. En effet, la recréation/ restauration d'un même chevelu hydrographique, dense, fortement interconnecté et spécifique aux têtes de bassin versant, ne paraît faisable que sur d'autres bassins aux mêmes configurations géographiques. En outre, le maintien de la variabilité hydraulique naturelle des cours d'eau demanderait de constituer des stocks d'eau en période de hautes eaux avec possibilité de les relâcher lors d'événements hydropluviométriques de plus faible périodicité.

- Les fonctions biologiques qui, avec les fonctions hydrologiques, participent fortement au choix des actions écologiques à mener. Elles sont déterminées au travers de l'étude de la capacité d'accueil des zones humides pour la flore et la faune (généralement déterminées au regard des espèces protégées présentes). La compensation effective de ces fonctions dépendra surtout de la disponibilité en zones humides dégradées pouvant être restaurées, de même nature et à proximité spatiale des sites impactés.

- Les fonctions biogéochimiques (épuration des eaux, etc.) étroitement associées à l'hydropériode. Leur restauration/recréation s'applique à toutes les zones à compenser, quel que soit leur type, et dépend aussi des surfaces que le maître d'ouvrage parviendra à acquérir ou à convertir à proximité de son projet. Certaines actions écologiques peuvent consister en l'aménagement de rives des cours d'eau, propices aux échanges nappesrivières, qui favorisent les phénomènes d'autoépuration. Ces fonctions paraissent moins contraignantes que les deux autres et peuvent éventuellement être superposées (mutualisées) à la fonction biologique, car les sites de compensation permettant de reconstituer des fonctions biologiques et microbiologiques pourront réduire certaines pressions anthropiques (dégradation bactérienne des éléments des pollutions agricoles, urbaines, etc.).

- Enfin, la valeur patrimoniale des milieux impactés, qui découle des sciences du paysage dans le sens de Donadieu (2012) et relève de la qualité paysagère d'un site. À ce titre, l'identification "d'unités de paysage opérationnel » (Operational Landscape Units) en territoire 
agricole néerlandais par Verhoeven et al. (2008) permet de faciliter la prise en compte simultanée des connexions hydrologiques (inondation, écoulement souterrain) et biologiques (dispersion, transport des organismes) et de restaurer ainsi un service écosystémique adéquat.

En outre, une grande incertitude existe quant aux résultats de la compensation. En France, les techniques qualifiées de génie écologique datent des années 1970 (Gosselin, 2004, 2008). Elles s'appuient sur les principes de l'ingénierie écologique résumés par Mitsch (2012) et qui résultent de trois décennies de recherches, de tests et de transferts (Barnaud et Chapuis, 2004). Les analyses montrent : (i) l'importance des délais de mise en œuvre de ces actions, variables selon l'écosystème considéré ; (ii) la non-garantie d'obtention des résultats escomptés et le risque élevé d'échec au regard d'un objectif initial trop ambitieux (Mistch et Jørgensen, 2004). Les chances de succès sont essentiellement conditionnées par une formulation précise des objectifs, une bonne connaissance de la nature et du fonctionnement hydrogéomorphologique des zones humides de compensation et des techniques d'ingénierie écologique mises en œuvre (Photo 2). Il est également indispensable de définir précisément les modalités d'évaluation de la réponse de compensation et de ses coefficients d'ajustement (risque d'échec, éloignement géographique, durée nécessaire à la restauration effective des fonctions, etc.), avant même le démarrage du chantier.

La question de l'incertitude quant à la pérennité des mesures se pose. La compensation par achat de crédits, qui commence à se développer en France, paraît très pragmatique mais devrait être davantage étudiée et réglementairement cadrée. En effet, les effets écologiques et sociologiques (positifs, négatifs) de cette approche, le problème de traçabilité des crédits ainsi que des dérives dans son application ont été mis en évidence ailleurs.

Concernant la sécurisation foncière des sites de compensation, la maîtrise foncière assure la pérennité de la compensation et augmente les chances de réussite des travaux de génie écologique envisagés. À défaut, la signature de contrats avec les propriétaires ou les bailleurs imposant le respect d'un cahier des charges est possible mais présente des difficultés quant à la pérennité des actions écologiques menées. À ce titre, la durée des contrats passés entre les maîtres d'ouvrage et les exploitants locaux via d'éventuels propriétaires fonciers doit être définie en fonction (i) du temps nécessaire à la restauration/renaturation des milieux, allant de quelques années s'il s'agit de mares, à quelques décennies, voire davantage, dans le cas de prairies oligotrophes; et/ou (ii) de l'ordre de grandeur des baux ruraux, pour les exploitations agricoles en activité, soit neuf ou dix-huit ans selon le type de travaux envisagé dans la parcelle concernée, ce qui peut s'avérer court en comparaison du temps nécessaire à la restauration des services écosytémiques. Dans ce cas particulier, la pérennité des mesures doit reposer sur la création d'une réserve supplémentaire de sites de compensation permettant de pallier l'échec des travaux de génie écologique, le désistement des propriétaires terriens ou l'arrêt des conventions signées entre le maître d'ouvrage et les gestionnaires ou exploitants du site.

De façon plus générale, l'expérience de Notre-Damedes-Landes a mis en évidence les causes d'une réelle résistance du monde agricole à la compensation : la création d'un aménagement de superficie importante tel qu'un aéroport se fait généralement sur des terres agricoles et nécessite d'affecter une partie supplémentaire de ces terres agricoles à la compensation écologique (même si un usage agricole reste possible sur ces territoires de compensation, photo 3). Ce constat milite pour que les besoins de compensation soient évalués, au même titre que l'emprise de l'aménagement, le plus en amont possible, lors des phases de conception du projet, et mentionnés dans les enquêtes ou débats publics. Faute de ces précautions initiales, on ne peut que tenter d'insérer les mesures de compensation dans l'environnement proche des emprises, avec des difficultés évidentes d'ordre écologique, foncier et socio-économique.

\section{Conclusion}

À bien des égards, le site de Notre-Dame-des-Landes est exemplaire: superficie importante des zones humides impactées, caractère " relique » d'un milieu agricole préservé du fait même de la mise en réserve depuis plusieurs décennies des terres pour l'aménagement envisagé (ce qui aurait pu permettre d'étudier et d'anticiper les mesures de compensation), caractère emblématique aux yeux des acteurs locaux. Mais notre expertise a également mis en évidence les limites d'un discours souvent théorique sur la compensation et les questions que soulève l'application sur le terrain de principes réglementaires tels que l'équivalence écologique, l'efficacité, la pérennité, etc. La compensation écologique s'inscrit dans une séquence "éviter, réduire, compenser » qui demande de s'assurer au préalable qu'il n'y a pas d'alternatives possibles. Mais la finalisation des mesures de compensation soulève divers problèmes concrets tels que la disponibilité et l'usage des terres à proximité de l'aménagement envisagé, le risque d'échec des travaux de génie écologique, la difficulté d'estimer les trajectoires temporelles des systèmes écologiques concernés, etc.

La première difficulté consiste en la sécurisation foncière de sites équivalents sur le plan écologique aux zones humides impactées, dans la région concernée. Où trouver les terres disponibles et comment contractualiser 


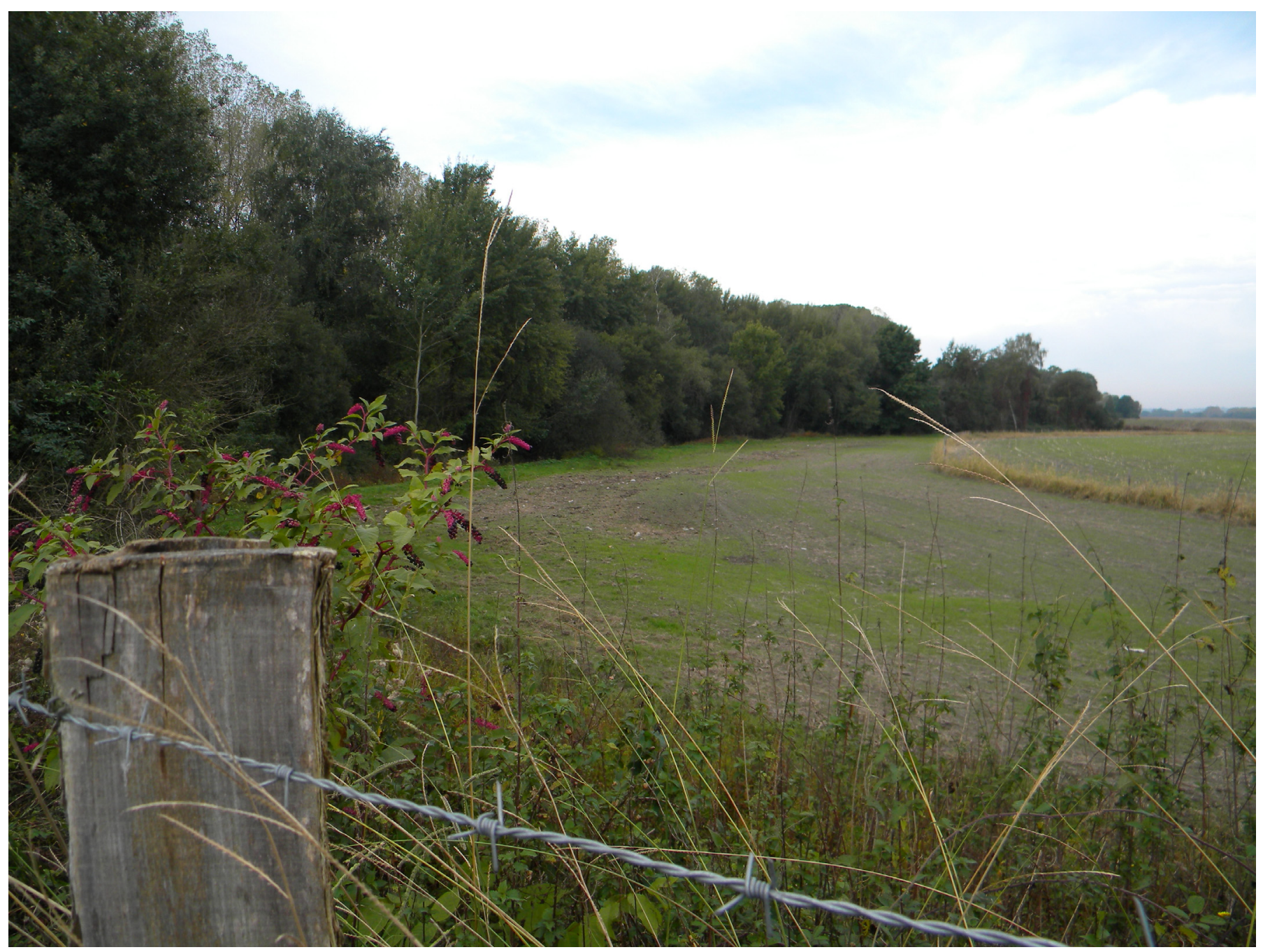

Photo 3. Site de compensation de l'autoroute A65 : conversion des cultures et restauration de prairies sur lesquelles un usage agricole est conservé (source : Nadia Moulin, Onema, 19 octobre 2012).

avec les propriétaires? En l'occurrence, l'aéroport de Notre-Dame-des-Landes est implanté dans une zone agricole d'élevage de bovins pour la production de lait et de viande, avec de nombreuses formations bocagères. L'agriculture y est dynamique, fortement consommatrice d'espaces mais soumise à la contrainte d'une urbanisation rapide. Dans ce contexte, la profession agricole s'est opposée au projet (Pélissié et al., 2013), en protestant notamment contre la «double peine» qui leur est imposée (perte de surfaces agricoles au profit de l'aéroport et de sa desserte routière, puis modification des pratiques culturales à cause des sites de compensation écologique). L'agriculture peut en effet apparaître comme une réserve foncière à mobiliser en cas de nécessité, avec les conséquences qui en découlent en termes de pertes d'emplois et de réduction du potentiel économique. Dans un tel contexte, passer des accords avec la profession agricole pour mener des actions à long terme est difficile, mais peut dépendre de l'évolution des pratiques en liaison avec les cours des produits agricoles. En tout état de cause, l'estimation et la localisation des superficies nécessaires à la compensation dès la conception des projets et surtout lors des enquêtes publiques apparaissent comme un impératif, au même titre que les mesures d'évitement et de réduction.

La deuxième difficulté tient aux limites des techniques du génie écologique. La compensation écologique s'est mise en place sur une idée simple : restaurer/recréer ailleurs ce qui est détruit ici. Mais sa mise en œuvre est complexe. Compenser à l'identique relève souvent de l'utopie, ce qui, pour tenir compte de la réglementation, amène à des propositions diverses dont le gain écologique est parfois limité. En outre, le risque d'échec des travaux de génie écologique est rarement pris en compte alors que les retours d'expérience indiquent que les objectifs sont rarement atteints. L'évaluation de 
l'efficacité des mesures de compensation nécessite la mise en œuvre simultanée de suivis de sites témoins et de sites de compensation pour vérifier le respect des objectifs initiaux. C'est le seul moyen de réajuster, si nécessaire, les actions écologiques mises en œuvre et de pérenniser les résultats. À cette fin, les protocoles de suivi doivent être définis très tôt, lors de la réalisation de l'état initial et avant même les réflexions préliminaires à la définition des actions à mener (Bazin et Barnaud, 2002). En outre et conformément aux engagements de la loi Grenelle II $^{9}$ (2010), il est pleinement justifié de saisir dans une base de données informatique les informations spécifiques aux sites de compensation (nature, situation géographique, modalités de sécurisation foncière, plan de gestion conservatoire, etc.), et de promouvoir la création d'observatoires de l'évolution des zones humides et des pratiques agricoles (comme les systèmes d'observation et d'expérimentation au long terme pour la recherche en environnement [SOERE] de l'Alliance nationale de recherche pour l'environnement [AllEnvi], 2013), notamment pour acquérir des références utiles sur ce type de mesures et de milieux.

La troisième difficulté relève de la prise en compte des aspects temporels. En pratique, les mesures de compensation peinent à être mises en œuvre dans les délais, $\mathrm{du}$ fait des questions foncières et sociétales qu'elles soulèvent, mais également du décalage temporel nécessaire à la reconstruction de ces écosystèmes, et à l'atteinte de leur plein fonctionnement écologique. Cela fragilise le processus global de compensation et oblige, pour les grands projets, à anticiper la perte éventuelle de sites de compensation par la recherche constante d'autres sites. D'autre part, les systèmes écologiques ne sont pas stationnaires, mais s'inscrivent sur des trajectoires spatiales et temporelles de long terme. Les changements climatiques à venir ( $c f$. projet Explore 2070 [MEDDE, 2012c]) risquent de modifier la répartition spatiale et la dynamique actuelle des zones humides. On ne peut donc pas se fixer logiquement pour objectif une compensation à l'identique. L'absence d'anticipation dans ce domaine est donc préjudiciable à l'esprit même de la compensation.

L'ensemble du débat sur la compensation devrait également se placer dans une dimension de développement durable et donc intégrer des aspects sociaux et économiques (Chéreau et al., 2013). Peut-on raisonner la compensation sans prendre en compte, par exemple, les impacts sur le développement économique des zones rurales ? Plus généralement, comment augmenter et/ou garantir l'acceptabilité sociale des mesures proposées?

\footnotetext{
9 Loi $\mathrm{n}^{\circ} 2010-788 \mathrm{du} 12$ juillet 2010 portant engagement national pour l'environnement (loi Grenelle II, 2010), http:/ / www.legifrance.gouv.fr/affichTexte.do?cidTexte= JORFTEXT000022470434.
}

N'existe-t-il pas une autre approche participative à développer pour ce faire? Les répercussions envisageables de l'émergence de marchés liés aux banques de compensation méritent également d'être examinées de près.

\section{Références}

Alliance nationale de recherche pour l'environnement (AllEnvi), 2013. Les Systèmes d'observation et d'expérimentation au long terme pour la recherche en environnement (SOERE), http:/ / www.allenvi.fr/groupes-transversaux/infrastructures-de-recherche/les-soere2/gouvernance-etfinancement.

Amigues, J.-P., Chevassus-au-Louis, B., 2011. Évaluer les services écologiques des milieux aquatiques: enjeux scientifiques, politiques et opérationnels, comprendre pour agir, Office national de l'eau et des milieux aquatiques (Onema).

Bakker, J.P., Berendse, F., 1999. Constraints in the restoration of ecological diversity in grassland and heathland communities, Trends in Ecology \& Evolution, 14, 63-68.

Barles, S., 2013. Socio-ecological trajectories. The urban dimension. Paris, 18th-20th centuries in Proceedings of ESE 2013 Conference "Ecological economics and institutional dynamics », Lille, June 18-21, European Society for Ecological Economics (ESEE).

Barnaud, G., Chapuis, J.-L., 2004. Ingénierie écologique et écologie de la restauration. Spécificités et complémentarités, Ingénierie - Eau, agriculture, territoires, $\mathrm{n}^{\circ}$ spécial, 123-138.

Barnaud, G., Coïc, B., 2011. Mesures compensatoires et correctives liées à la destruction des zones humides. Revue bibliographique et analyse critique des méthodes. Rapport final, Onema, Muséum national d'histoire naturelle, Paris.

Bazin, P., Barnaud, G., 2002. Du suivi à l'évaluation. À la recherche d'indicateurs opérationnels en écologie de la restauration, in Chapuis, J.-L., Décamps, H., Barnaud, G. (Eds), Actes du colloque du Programme national de recherche "Recréer la nature», Grenoble, 11-13 septembre 2001, Revue d'écologie - la Terre et la Vie, supplément n 9, 201-224.

Bendor, T., Riggsbee, A., Howard, G., 2009. Mitigation bankers say new rule heightens old conflicts: survey, Ecosystem Marketplace.

Benoît, M., Rizzo, D., Marraccini, E., Rapey, H., Thenail, C., Moonen, A.C., Lardon, S., Galli, M., Caron, P., Silvestri, N., Bonari, E., 2012. Landscape agronomy. Conceptualizing a new research field in landscape ecology, Landscape Ecology, $10,27,1385-1394$.

Bernard, P., 1994. Les zones humides. Rapport d'évaluation, Comité interministériel de l'évaluation des politiques publiques, Premier Ministre, Commissariat au Plan, Paris, La Documentation française.

Blandin, P., Lamotte, M., 1984. Écologie des systèmes et aménagement. Fondements théoriques et principes méthodologiques, in Lamotte, M. (Ed.), Fondements rationnels de l'aménagement d'un territoire, Paris, Masson, 139-162.

Burel, F., Baudry, J., 1999. Écologie du paysage. Concepts, méthodes et applications, Paris, Tec \& Doc.

Chéreau, C., Brévan, C., Kbaier, R., 2013. Transfert de l'aéroport de Nantes Atlantique. Rapport de la Commission du 
dialogue, Paris, ministère de l'Écologie, du Développement durable et de l'Énergie.

Clean Water Act, 1972. Federal water pollution control act amendment of 1972, to amend the federal water pollution control act, enacted by the 92th US Congress, Public Law 92-500, 86, 816, October 18th 1972, http:/ /www.gpo.gov/fdsys/pkg/ STATUTE-86/pdf/STATUTE-86-Pg816.pdf.

Committee on Mitigating Wetland Losses, Board on Environmental Studies and Toxicology, Water Science and Technology Board, Division on Earth and Life Studies, National Research Council, 2001. Compensating for wetland losses under the clean water act, Washington, D.C., National Academy Press.

Commissariat général au développement durable (CGDD), Direction de l'eau et de la biodiversité (DEB), 2013. Lignes directrices nationales sur la séquence éviter, réduire et compenser les impacts sur les milieux naturels, Paris, ministère de l'Écologie, du Développement durable et de l'Énergie (MEDDE), http://www.developpement-durable.gouv.fr/ IMG/pdf/Ref_-_Lignes_directrices.pdf.

Comité d'orientation pour des pratiques agricoles respectueuses de l'environnement (CORPEN), 2007. Les fonctions environnementales des zones tampons. Les bases scientifiques et techniques des fonctions de production des eaux, Paris, ministère de l'Écologie, du Développement durable et de l'Aménagement du Territoire, Ministère de l'Agriculture et de la Pêche.

Costanza, R., d'Arge, R., de Groot, R., Farber, S., Grasso, M., Hannon, B., Limburg, K., , Naeem, S., O'Neill, R.V., Paruelo, J., Raskin, R.G., Sutton, P. 1997. The value of the world's ecosystem services and natural capital, Nature, 387, 253-260.

Dahl, T.E., 1990.Wetlands losses in the United States: 1790's to 1980 's. Report to Congress, US Department of Interior, Fish and Wildlife Service, Washington D.C., https:// www.fws.gov/wetlands/Documents/Wetlands-Lossesin-the-United-States-1780s-to-1980s.pdf.

Donadieu, P., 2012. Sciences du paysage. Entre théories et pratiques, Paris, Tec \& Doc.

Erwin, K.L., 2009. Wetlands and global climate change. The role of wetland restoration in a changing world, Wetlands Ecology and Management, 17, 71-84, doi: 10.1007/s11273-0089119-1.

Forget, G., Carreau, C., Le Cœur, D., Bernez, I., 2013. Ecological restoration of headwaters in a rural landscape (Normandy, France). A passive approach taking hedge networks into account for riparian tree recruitment, Restoration Ecology, 21, 1, 96-104.

Forman, R.T.T., Godron, M., 1986. Landscape ecology, New York, John Wiley.

GAO (United States Governement Accountability Office), 2001. Wetlands protection. Assessments needed to determine effectiveness of in-lieu-fee mitigation.. Report to congressional requesters, United States Government Accountability Office, GAO-01-325.

GAO (United States Governement Accountability Office), 2005. Wetlands protection. Corps of engineers does not have an effective oversight approach to ensure that compensatory mitigation is occurring. Report to the Ranking Democratic Member, Committee on Transportation and Infrastructure,
House of Representatives, United States Government Accountability Office, GAO, 05, 898.

Geniaux, G., 2002. Le mitigation banking. Un mécanisme décentralisé au service des politiques de no net loss, in Centre de recherches interdisciplinaires en droit de l'environnement, de l'aménagement et de l'urbanisme (Ed.), Les difficultés de mise en cuvre de la directive Habitats sous les regards croisés de sociologues, juristes, économistes et géographes, Actes et communications de l'Inra, 19, 57-71.

Gosselin, F., 2004. Intégrer recherche scientifique en écologie et gestion dans le cadre de l'ingénierie écologique. Intérêts et limites, Ingénierie - Eau, agriculture, territoires, $\mathrm{n}^{\circ}$ spécial, 113-120.

Gosselin, F., 2008. Redefining ecological engineering to promote its integration with sustainable development and tighten its links with the whole of ecology, Ecological Engineering, 32, 199-205.

Joyce, C.B., 2014. Ecological consequences and restoration potential of abandoned wet grasslands, Ecological Engineering, 66, 91-102.

Lefeuvre, J.-C., 1990. De la protection de la nature à la gestion du patrimoine naturel, in Jeudy, P.-H. (Ed.), Patrimoines en folie, Paris, Éditions de la Maison des sciences de l'homme, 29-75.

Lévêque, C., Van der Leeuw, S.E. (Eds), 2003. Quelles natures voulons-nous? Pour une approche socio-écologique du champ de l'environnement, Paris, Elsevier.

Lévêque, C., 2013. L'écologie est-elle encore scientifique?, Versailles, Quæ.

Loreau, M., Mouquet, N., Gonzalez, A., 2003. Biodiversity as spatial insurance in heterogeneous landscapes, Proceedings of the National Academy of Sciences, 100, 12765-12770, http: / / www.pnas.org/content/100/22/12765.full.pdf.

Madsen, B., Caroll, N., Brands, M.K., 2010. State of biodiversity markets report. Offset and compensation programs worldwide, Washington (DC), Forest Trends.

Madsen, B., Kandy, D., Bennett, G., 2011. Update. State of biodiversity markets, Washington (DC), Forest Trends.

Maron, M., Hobbs, R.J., Moilanen, A., Matthews, J.W., Christie, K., Gardner, T.A., Keith, D.A., Lindenmayer, D.B., McAlpine, C.A., 2012. Faustian bargains? Restoration realities in the context of biodiversity offset policies, Biological Conservation, 155, 141-148.

Matthews, J.W., Endress, A.G., 2008. Performance criteria, compliance success, and vegetation development in compensatory mitigation wetlands, Environmental Management, 41, 130-141.

Millennium Ecosystem Assessment (Ed.), 2005. Ecosystems and human well-being. Synthesis, London, Island Press.

Ministère de l'Écologie, du Développement durable et de l'Énergie (MEDDE), 2012a. Doctrine relative à la séquence éviter, réduire et compenser les impacts sur le milieu naturel.

Ministère de l'Écologie, du Développement durable et de l'Énergie (MEDDE), 2012b. Explore 2070. Vulnérabilité des milieux aquatiques et de leurs écosystèmes. Étude des zones humides, http://www.developpement-durable.gouv.fr/ IMG/pdf/Etude_sur_les_zones_humides.pdf.

Ministère de l'Écologie, du Développement durable et de l'Énergie (MEDDE), 2012c. Explore 2070. Eau et changement climatique, quelles stratégies d'adaptation possibles? 
Mitsch, W.J., Wilson, R.F., 1996. Improving the success of wetland creation and restoration with know-how, time, and self-design, Ecological Applications, 6, 77-83.

Mitsch, W.J., Jørgensen, S.E., 2004. Ecological engineering and ecosystem restoration, New York, John Wiley.

Mitsch, W.J., 2012. What is ecological engineering?, Ecological Engineering, 45, 5-12.

Moilanen, A., Van Teeffelen, A.J.A., Ben-Haim, Y., Ferrier, S., 2009. How much compensation is enough? A framework for incorporating uncertainty and time discounting when calculating offset ratios for impacted habitat, Restoration Ecology, 17, 470-478.

Morandeau, D., Vilaysack, D., 2012. La compensation des atteintes à la biodiversité à l'étranger. Étude de parangonnage, Commissariat général au développement durable, Service de l'économie, de l'évaluation et de l'intégration du développement durable (CGDD, SEEIDD), Études et documents, 68, 1-134.

Moreno-Mateos, D., Power, M-E., Comín, F-A., Yockteng, R., 2012. Structural and functional loss in restored wetland ecosystems, PLoS Biology, 10, 1-9, doi: 10.1371/ journal.pbio.1001247.

Morera, R., 2011. L'assèchement des marais en France au XVII ${ }^{e}$ siècle, Presses universitaires de Rennes.

Muller, S., Dutoit, T., Alard, D., Grevilliot, F., 1998. Restoration and rehabilitation of species rich-grasslands in France. A review, Restoration Ecology, 6, 94-101.

Parlement européen et Conseil de l'Union européenn, 2000. Directive 2000/60/CE établissant un cadre pour une politique communautaire dans le domaine de l'eau, 23 octobre 2000.

Pélissié, D., Nil, D., Stephan, J.M., 2013. Projet d'aéroport de Notre-Dame-des-Landes. Expertise de l'impact sur l'agriculture, Paris, ministère de l'Agriculture, de l'Agroalimentaire et de la Forêt.

Pywell, R.F., Bullock, J.M., Hopkins, A., Walker, K.J., Sparks, T.H., Burke, M.J.W., Peel, S. , 2002. Restoration of speciesrich grassland on arable land. Assessing the limiting processes using a multi-site experiment, Journal of Applied Ecology, 39, 294-309.

Quétier, F., Lavorel, S., 2011. Assessing ecological equivalence in biodiversity offset schemes. Key issues and solutions, Biological Conservation, 144, 2991-2999.

Quétier, F., Quenouille, B., Schwoertzig, E., Gaucherand, S., Lavorel, S., Thiévent, P., 2012. Les enjeux de l'équivalence écologique pour la conception et le dimensionnement de mesures compensatoires d'impacts sur la biodiversité et les milieux naturels, Sciences Eaux $\mathcal{E}$ Territoires, article hors-série, http: / / www.set-revue.fr/les-enjeux-de-l-equivalenceecologique-pour-la-conception-et-le-dimensionnement-demesures-compensat.
Robb, J.T., 2002. Assessing wetland compensatory mitigation sites to aid in establishing mitigation ratios, Wetlands, 22, 435-440.

Robson, B.J., Mitchell, B.D., Chester, E.T., 2011. An outcomebased model for predicting recovery pathways in restored ecosystems. The recovery cascade model, Ecological Engineering, 37, 1379-1386.

Ruhl, J.B., Salzman, J., 2006. The effects of wetland mitigation banking on people, National Wetlands Newsletter, 28, 9-14.

Salzman, J., Ruhl, J.B., 2005. « No net-loss ». Instrument choice in wetlands protection, in Freeman, J., Kolstad, C.D. (Eds), Moving to markets in environmental regulation. Lessons from twenty years of experience. Oxford/New York, Oxford University Press, 323-350.

Schéma directeur d'aménagement et de gestion des eaux (SDAGE) du bassin Loire-Bretagne, 2010-2015.

Scemama, P., Level, H., 2013. L'émergence du marché de la compensation des zones humides aux États-Unis : impacts sur les modes d'organisation et les caractéristiques des transactions, Revue d'économie politique, 6, 123, 893-924.

Tiner, R. (Ed.), 2002. Watershed-based wetland planning and evaluation. A collection of papers from the wetland Millennium event. Proceedings of a symposium at the Wetland Millennium Event, Quebec City, Quebec, Canada, August 6-12, 2000.

Turner, R.E., Redmond, A.M., Zedler, J.B., 2001. Count it by acre or function. Mitigation adds up to net loss of wetlands, National Wetlands Newsletter, 23, 5-6.

Vécrin, M.-P., Muller, S., 2003. Top-soil translocation as a technique in the re-creation of species-rich meadows, Applied Vegetation Science, 6, 271-278.

Vécrin, M.-P., Grévilliot, F., Muller, S., 2007. The contribution of persistent soil seed banks and flooding to restoration of alluvial meadows, Journal for Nature Conservation, 15, 59-69.

Verhoeven, J.T.A., Soons, M.B., Janssen, R., Omtzigt, N., 2008. An operational landscape unit approach for identifying key landscape connections in wetland restoration, Journal of Applied Ecology, 45, 1496-1503.

Whigham, D.F., 1999. Ecological issues related to wetland preservation, restoration, creation and assessment, The Science of the Total Environment, 240, 31-40.

Zedler, J.B., Callaway, J.C., 1999. Tracking wetland restoration. Do mitigation sites follow desired trajectories?, Restoration Ecology, 7, 69-73.

Zedler, J.B., Doherty, J.M., Miller., N.A., 2012. Shifting restoration policy to address landscape change, novel ecosystems and monitoring, Ecology and Society, 17, 4, article 36, http:/ /dx.doi.org/10.5751/ES-05197-170436. 